\title{
LEFT-ORDERABLE COMPUTABLE GROUPS
}

\author{
MATTHEW HARRISON-TRAINOR
}

\begin{abstract}
Downey and Kurtz asked whether every orderable computable group is classically isomorphic to a group with a computable ordering. By an order on a group, one might mean either a left-order or a bi-order. We answer their question for left-orderable groups by showing that there is a computable left-orderable group which is not classically isomorphic to a computable group with a computable left-order. The case of bi-orderable groups is left open.
\end{abstract}

\section{INTRODUCTION}

A left-ordered group is a group $\mathcal{G}$ together with a linear order $\leq$ such that if $a \leq b$, then $c a \leq c b$. $\mathcal{G}$ is right-ordered if instead whenever $a \leq b, a c \leq b c$, and bi-ordered if $\leq$ is both a left-order and a right-order. A group which admits a leftordering is called left-orderable, and similarly for right- and bi-orderings. A group is left-orderable if and only if it is right-orderable. Some examples of bi-orderable groups include torsion-free abelian groups and free groups [Shi47, Vin49, Ber90. The group $\left\langle x, y: x^{-1} y x=y^{-1}\right\rangle$ is left-orderable but not bi-orderable. For a reference on orderable groups, see KM96.

In this paper, we will consider left-orderable computable groups. A computable group is a group with domain $\omega$ whose group operation is given by a computable function $\omega \times \omega \rightarrow \omega$. Downey and Kurtz DK86] showed that a computable group, even a computable abelian group, which is orderable need not have a computable order. If a computable group does admit a computable order, we say that it is computably orderable. Of course, by the low basis theorem, every orderable computable group has a low ordering.

For an abelian group, any left-ordering (or right-ordering) is a bi-ordering. An abelian group is orderable if and only if it is torsion-free. Given a computable torsion-free abelian group $\mathcal{G}$, Dobritsa Dob83] showed that there is another computable group $\mathcal{H}$, which is classically isomorphic to $\mathcal{G}$, which has a computable $\mathbb{Z}$-basis. Note that $\mathcal{H}$ need not be computably isomorphic to $\mathcal{G}$. Solomon Sol02 noted that a $\mathbb{Z}$-basis for a torsion-free abelian group computes an ordering of that group. Hence every orderable computable abelian group is classically isomorphic to a computably orderable group.

Downey and Kurtz asked whether this is the case even for non-abelian groups:

Question 1 (Downey and Kurtz [DR00]). Is every orderable computable group classically isomorphic to a computably orderable group?

If one takes "orderable" to mean "left-orderable" then we give a negative answer to this question. (We leave open the question for bi-orderable groups.)

The author was partially supported by the Berkeley Fellowship and NSERC grant PGSD3454386-2014. The author would like to thank Antonio Montalbán for reading and commenting on a draft of this article. 
Theorem 2. There is a computable left-orderable group which has no presentation with a computable left-ordering.

Our strategy is to build a group

$$
\mathcal{G}=\mathcal{N} \rtimes \mathcal{H} / \mathcal{R}
$$

and code information into the finite orbits of certain elements of $\mathcal{N}$ under inner automorphisms given by conjugating by elements of $\mathcal{H} / \mathcal{R}$. This strategy cannot work to build a bi-orderable group, as in a bi-orderable group there is no generalized torsion-i.e., no product of conjugates of a single element can be equal to the identity - and hence no inner automorphism has a non-trivial finite orbit. We leave open the case of bi-orderable groups.

\section{Notation}

We will use caligraphic letter such as $\mathcal{G}, \mathcal{N}$, and $\mathcal{H}$ to denote groups. For free groups, we will use upper case latin letters such as $A, B, C, U, V$, and $W$ to denote words, while using lower case letters such as $a, b$, and $c$ to denote letter variables. We use $\varepsilon$ for the empty word, 0 for the identity element of abelian groups, and 1 for the identity element of non-abelian groups (except for free groups, where we use $\varepsilon)$.

\section{The Construction}

Fix $\psi$ a partial computable function which we will specify later (see Definition 8). Let $p_{i}, q_{i}$, and $r_{i}$ be a partition of the odd primes into three lists 1 Let $\mathcal{H}$ be the free abelian group on $\alpha_{i}, \beta_{i}$, and $\gamma_{i}$ for $i \in \omega$. We write $\mathcal{H}$ additively. Let $\mathcal{R}$ be the set of relations

$$
\mathcal{R}=\left\{\mathcal{R}_{i, t}: \psi_{\text {at } t}(i) \downarrow\right\}
$$

where

$$
\mathcal{R}_{i, t}=\left\{\begin{array}{ll}
p_{i}^{t} \alpha_{i}=q_{i}^{t} \beta_{i} & \text { if } \psi_{\text {at } t}(i)=0 \\
p_{i}^{t} \alpha_{i}=-q_{i}^{t} \beta_{i} & \text { if } \psi_{\text {at } t}(i)=1
\end{array} .\right.
$$

By $\psi_{\text {at } t}(i)=0$, we mean that the computation $\psi(i)$ has converged exactly at stage $t$ (but not before) and equals zero.

The idea is that these relations force, for any ordering $\leq$ on $\mathcal{H} / \mathcal{R}$, that if $\psi(i)=0$ then $\alpha_{i}>0 \Longleftrightarrow \beta_{i}>0$ (and if $\psi(i)=1$ then $\alpha_{i}>0 \Longleftrightarrow \beta_{i}<0$ ). The strategy is, in a very general sense, to use $\psi$ to diagonalize against computable orderings of $\mathcal{H} / \mathcal{R}$. The semidirect product will add enough structure to allow us to find $\alpha_{i}$ and $\beta_{i}$ within a computable copy of $\mathcal{G}$. (One cannot find $\alpha_{i}$ and $\beta_{i}$ within a copy of $\mathcal{H} / \mathcal{R}$, since $\mathcal{H} / \mathcal{R}$ is a torsion-free abelian group.) Note that

$$
\mathcal{H} / \mathcal{R}=\left(\bigoplus_{i}\left\langle\alpha_{i}, \beta_{i}\right\rangle / \mathcal{R}_{i}\right) \oplus\left(\bigoplus\left\langle\gamma_{i}\right\rangle\right)
$$

where $\mathcal{R}_{i}=\mathcal{R}_{i, t}$ if $\psi_{\text {at } t}(i) \downarrow$ for some $t$, or no relation otherwise. Define

$$
\begin{array}{lll}
\mathcal{V}_{i}=\mathcal{R} \cup\left\{p_{i} \alpha_{i}=0\right\} & \mathcal{W}_{i}=\mathcal{R} \cup\left\{q_{i} \beta_{i}=0\right\} & \mathcal{X}_{i}=\mathcal{R} \cup\left\{r_{i} \gamma_{i}=0\right\} \\
\mathcal{Y}_{i}=\mathcal{R} \cup\left\{\alpha_{i}=\gamma_{i}\right\} & \mathcal{Z}_{i}=\mathcal{R} \cup\left\{\beta_{i}=\gamma_{i}\right\} . &
\end{array}
$$

\footnotetext{
${ }^{1}$ We use the fact that 2 does not appear in these lists in Lemma 22
} 
Let $\mathcal{N}$ be the free (non-abelian) group on the letters

$$
\begin{array}{r}
\left\{u_{i}: i \in \omega\right\} \cup\left\{v_{i, g}: g \in \mathcal{H} / \mathcal{V}_{i}, i \in \omega\right\} \cup\left\{w_{i, g}: g \in \mathcal{H} / \mathcal{W}_{i}, i \in \omega\right\} \\
\cup\left\{x_{i, g}: g \in \mathcal{H} / \mathcal{X}_{i}, i \in \omega\right\} \cup\left\{y_{i, g}: g \in \mathcal{H} / \mathcal{Y}_{i}, i \in \omega\right\} \cup\left\{z_{i, g}: g \in \mathcal{H} / \mathcal{Z}_{i}, i \in \omega\right\} .
\end{array}
$$

Let $\mathcal{G}=\mathcal{N} \rtimes(\mathcal{H} / \mathcal{R})$, with $g \in \mathcal{H} / \mathcal{R}$ acting on $\mathcal{N}$ via the automorphism $\varphi_{g}$ as follows:

$$
\begin{aligned}
& \varphi_{g}\left(u_{i}\right)=u_{i} \quad \varphi_{g}\left(v_{i, h}\right)=v_{i, \bar{g}+h} \quad \varphi_{g}\left(w_{i, h}\right)=w_{i, \bar{g}+h} \\
& \varphi_{g}\left(x_{i, h}\right)=x_{i, \bar{g}+h} \quad \varphi_{g}\left(y_{i, h}\right)=y_{i, \bar{g}+h} \quad \varphi_{g}\left(z_{i, h}\right)=z_{i, \bar{g}+h} .
\end{aligned}
$$

Here, $\bar{g}$ is the image of $g$ under the quotient map $\mathcal{H} / \mathcal{R} \rightarrow \mathcal{H} / \mathcal{V}_{i}$ (or $\mathcal{H} / \mathcal{W}_{i}, \mathcal{H} / \mathcal{X}_{i}$, etc.). Recall that the semidirect product $\mathcal{G}=\mathcal{N} \rtimes(\mathcal{H} / \mathcal{R})$ is the group with underlying set $\mathcal{N} \times(\mathcal{H} / \mathcal{R})$ with group operation

$$
(n, g)(m, h)=\left(n \varphi_{g}(m), g+h\right) .
$$

Note that $\varphi_{g}$ permutes the letters of $\mathcal{N}$, and so given a word $A \in \mathcal{N}, \varphi_{g}(A)$ is a word of the same length as $A$. We write $\mathcal{G}$ multiplicatively.

Lemma 3. $\mathcal{H} / \mathcal{R}$ has a computable presentation.

Proof. It suffices to show that we can decide whether or not a relation of the form

$$
\sum_{i=1}^{k} \ell_{i} \alpha_{i}+\sum_{i=1}^{k} m_{i} \beta_{i}+\sum_{i=1}^{k} n_{i} \gamma_{i}=0
$$

holds. This sum is equal to zero if and only if each $n_{i}=0$ and for each $i$ we have $\ell_{i} \alpha_{i}+m_{i} \beta_{i}=0$. So it suffices to decide, for a given $\ell$ and $m$ in $\mathbb{Z}$, whether $\ell \alpha_{i}=m \beta_{i}$.

Looking at $\mathcal{R}, \ell \alpha_{i}=m \beta_{i}$ if and only if either

(1) for some $t, \psi_{\text {at } t}(i)=0$ and there is $s \in \mathbb{Z}$ such that $\ell=s p_{i}^{t}$ and $m=s q_{i}^{t}$ or

(2) for some $t, \psi_{\text {at } t}(i)=1$ and there is $s \in \mathbb{Z}$ such that $\ell=s p_{i}^{t}$ and $m=-s q_{i}^{t}$. If $t>|\ell|$ or $t>|m|$ then neither of these can hold. So we just need to check, for each $t \leq|\ell|,|m|$, whether $\psi_{\text {at } t}(i)$ converges.

Lemma 4. $\mathcal{G}$ has a computable presentation.

Proof. We just need to check that $\mathcal{H} / \mathcal{V}_{i}, \mathcal{H} / \mathcal{W}_{i}$, and so on have computable presentations. We will see that the embeddings of the computable presentation (from the previous lemma) of $\mathcal{H} / \mathcal{R}$ into these presentations are computable. Then the action $\varphi$ of $\mathcal{H} / \mathcal{R}$ on $\mathcal{N}$ is computable. We can construct a computable presentation of $\mathcal{G}$ as the semidirect product $\mathcal{N} \rtimes(\mathcal{H} / \mathcal{R})$ under this computable action.

We need to decide whether in $\mathcal{H} / \mathcal{V}_{i}$ we have a relation

$$
\sum_{j=1}^{k} \ell_{j} \alpha_{j}+\sum_{j=1}^{k} m_{j} \beta_{j}+\sum_{j=1}^{k} n_{j} \gamma_{j}=0 .
$$

It suffices to decide, for a given $j$, whether

$$
\ell \alpha_{j}+m \beta_{j}+n \gamma_{j}=0
$$

If $j \neq i$, this is just as in the previous lemma. Otherwise, this holds if and only if $p_{i}$ divides $\ell, q^{t}$ divides $m$ for some $t$ with $\psi_{\text {at } t}(i) \downarrow$, and $n=0$. As before, we can check this computably.

The other cases - for $\mathcal{H} / \mathcal{W}_{i}, \mathcal{H} / \mathcal{X}_{i}$, and so on-are similar. 
Lemma 5. $\mathcal{H} / \mathcal{R}$ is a torsion-free abelian group.

Proof. $\mathcal{H} / \mathcal{R}$ is abelian as $\mathcal{H}$ was abelian. Recall that

$$
\mathcal{H} / \mathcal{R}=\left(\bigoplus_{i}\left\langle\alpha_{i}, \beta_{i}\right\rangle / \mathcal{R}_{i}\right) \oplus\left(\bigoplus_{i}\left\langle\gamma_{i}\right\rangle\right)
$$

where $\mathcal{R}_{i}=\mathcal{R}_{i, t}$ if $\psi_{\text {at } t}(i) \downarrow$ for some $t$, or no relation otherwise. So it suffices to show that $\left\langle\alpha_{i}, \beta_{i}\right\rangle / \mathcal{R}_{i}$ is torsion-free. If $\mathcal{R}_{i}$ is no relation, then this is obvious. So now suppose that $\psi_{\text {at } t}(i)=0$ and that

$$
k\left(m \alpha_{i}+n \beta_{i}\right)=\ell\left(p_{i}^{t} \alpha_{i}-q_{i}^{t} \beta_{i}\right)
$$

in $\left\langle\alpha_{i}, \beta_{i}\right\rangle$. Since $\mathcal{H}$ is torsion-free, we may assume that $\operatorname{gcd}(k, \ell)=1$. Then $k m=\ell p_{i}^{t}$ and $k n=-\ell q_{i}^{t}$. So we must have $k= \pm 1$, in which case $m \alpha_{i}+n \beta_{i}$ is already zero in $\left\langle\alpha_{i}, \beta_{i}\right\rangle / \mathcal{R}_{i}$. Thus $\left\langle\alpha_{i}, \beta_{i}\right\rangle / \mathcal{R}_{i}$ is torsion-free. The case where $\psi_{\text {at } t}(i)=1$ is similar.

Lemma 6. $\mathcal{G}$ is left-orderable.

Proof. Since $\mathcal{H} / \mathcal{R}$ is a torsion-free abelian group, it is bi-orderable. $\mathcal{N}$ is biorderable as it is a free group. Then by the following claim, $\mathcal{G}$ is left-orderable (see Theorem 1.6.2 of [KM96]).

Claim 7. Let $\mathcal{A} \rtimes \mathcal{B}$ be a semi-direct product of left-orderable groups. Then $\mathcal{A} \rtimes \mathcal{B}$ is left-orderable.

Proof. Let $\varphi$ be the action of $\mathcal{B}$ on $\mathcal{A}$. Let $\leq_{\mathcal{A}}$ and $\leq_{\mathcal{B}}$ be left-orderings on $\mathcal{A}$ and $\mathcal{B}$ respectively. Define $\leq$ on $\mathcal{A} \rtimes \mathcal{B}$ as follows: $(a, b) \leq\left(a^{\prime}, b^{\prime}\right)$ if $b<_{\mathcal{B}} b^{\prime}$ or $b=b^{\prime}$ and $\varphi_{b^{-1}}(a) \leq_{\mathcal{A}} \varphi_{b^{-1}}\left(a^{\prime}\right)$. This is clearly reflexive and symmetric. We must show that it is transitive and a left-ordering.

Suppose that $(a, b) \leq\left(a^{\prime}, b^{\prime}\right) \leq\left(a^{\prime \prime}, b^{\prime \prime}\right)$. Then $b \leq_{\mathcal{B}} b^{\prime} \leq_{\mathcal{B}} b^{\prime \prime}$. If $b<_{\mathcal{B}} b^{\prime \prime}$, then $(a, b) \leq\left(a^{\prime \prime}, b^{\prime \prime}\right)$, so suppose that $b=b^{\prime}=b^{\prime \prime}$. Then

$$
\varphi_{b^{-1}}(a) \leq_{\mathcal{A}} \varphi_{b^{-1}}\left(a^{\prime}\right)=\varphi_{b^{\prime-1}}\left(a^{\prime}\right) \leq_{\mathcal{A}} \varphi_{b^{\prime-1}}\left(a^{\prime \prime}\right)=\varphi_{b^{-1}}\left(a^{\prime \prime}\right) .
$$

So $\varphi_{b^{-1}}(a) \leq_{\mathcal{A}} \varphi_{b^{-1}}\left(a^{\prime \prime}\right)$ and so $(a, b) \leq\left(a^{\prime \prime}, b^{\prime \prime}\right)$. Thus $\leq$ is transitive.

Given $(a, b) \leq\left(a^{\prime}, b^{\prime}\right)$ we must show that $\left(a^{\prime \prime}, b^{\prime \prime}\right)(a, b) \leq\left(a^{\prime \prime}, b^{\prime \prime}\right)\left(a^{\prime}, b^{\prime}\right)$. We have that

$$
\left(a^{\prime \prime}, b^{\prime \prime}\right)(a, b)=\left(a^{\prime \prime} \varphi_{b^{\prime \prime}}(a), b^{\prime \prime} b\right) \text { and }\left(a^{\prime \prime}, b^{\prime \prime}\right)\left(a^{\prime}, b^{\prime}\right)=\left(a^{\prime \prime} \varphi_{b^{\prime \prime}}\left(a^{\prime}\right), b^{\prime \prime} b^{\prime}\right) .
$$

If $b<_{\mathcal{B}} b^{\prime}$, then $b^{\prime \prime} b<_{\mathcal{B}} b^{\prime \prime} b^{\prime}$, and so $\left(a^{\prime \prime}, b^{\prime \prime}\right)(a, b) \leq\left(a^{\prime \prime}, b^{\prime \prime}\right)\left(a^{\prime}, b^{\prime}\right)$. Otherwise, if $b=b^{\prime}$ and $\varphi_{b^{-1}}(a) \leq_{\mathcal{A}} \varphi_{b^{-1}}\left(a^{\prime}\right)$, then $b^{\prime \prime} b=b^{\prime \prime} b^{\prime}$ and

$$
\begin{aligned}
\varphi_{\left(b^{\prime \prime} b\right)^{-1}}\left(a^{\prime \prime} \varphi_{b^{\prime \prime}}(a)\right) & =\varphi_{\left(b^{\prime \prime} b\right)^{-1}}\left(a^{\prime \prime}\right) \varphi_{b^{-1}}(a) \\
& \leq_{\mathcal{A}} \varphi_{\left(b^{\prime \prime} b\right)^{-1}}\left(a^{\prime \prime}\right) \varphi_{b^{-1}}\left(a^{\prime}\right) \\
& =\varphi_{\left(b^{\prime \prime} b\right)^{-1}}\left(a^{\prime \prime} \varphi_{b^{\prime \prime}}\left(a^{\prime}\right)\right) .
\end{aligned}
$$

So $\left(a^{\prime \prime}, b^{\prime \prime}\right)(a, b) \leq\left(a^{\prime \prime}, b^{\prime \prime}\right)\left(a^{\prime}, b^{\prime}\right)$.

Note that if $\leq$ is any left-ordering on $\mathcal{G}$, if $\psi_{\text {at } t}(i)=0$ then $\left(\varepsilon, \alpha_{i}\right)>1$ if and only if $\left(\varepsilon, \beta_{i}\right)>1$. On the other hand, if $\psi_{\text {at } t}(i)=1$ then $\left(\varepsilon, \alpha_{i}\right)>1$ if and only if $\left(\varepsilon, \beta_{i}\right)<1$. Later, in Definition 18, we will define existential formulas Same $(i)$ and Different $(i)$ (with no parameters) in the language of ordered groups. We would like to have that for any left-ordering $\leq$ on $\mathcal{G},(\mathcal{G}, \leq) \models \operatorname{Same}(i)$ if and only if $\left(\varepsilon, \alpha_{i}\right)>1 \Longleftrightarrow\left(\varepsilon, \beta_{i}\right)<1$, and $(\mathcal{G}, \leq) \models \operatorname{Different}(i)$ if and only if 
$\left(\varepsilon, \alpha_{i}\right)>1 \Longleftrightarrow\left(\varepsilon, \beta_{i}\right)<1$. We will not quite get this for every ordering $\leq$, but this will be true for those against which we want to diagonalize (see Lemma 9).

Definition 8. Fix a list $\left(\mathcal{F}_{i}, \leq_{i}\right)_{i \in \omega}$ of the (partial) computable structures in the language of ordered groups. Let $\psi$ be a partial computable function with $\psi(i)=0$ if $\left(\mathcal{F}_{i}, \leq_{i}\right) \models \operatorname{Different}(i)$ and $\psi(i)=1$ if $\left(\mathcal{F}_{i}, \leq_{i}\right) \models \operatorname{Same}(i)$. It is possible, a priori, that we have both $\left(\mathcal{F}_{i}, \leq_{i}\right) \models \operatorname{Same}(i)$ and $\left(\mathcal{F}_{i}, \leq_{i}\right) \models \operatorname{Different}(i)$; in this case, let $\psi(i)$ be defined according to whichever existential formula we find to be true first.

In fact, we will discover from the following lemma that we cannot have both $\left(\mathcal{F}_{i}, \leq_{i}\right) \models \operatorname{Same}(i)$ and $\left(\mathcal{F}_{i}, \leq_{i}\right) \models \operatorname{Different}(i)$.

Lemma 9. Fix $i$. Suppose that $\mathcal{F}_{i}$ is isomorphic to $\mathcal{G}$ and $\leq_{i}$ is a computable left-ordering of $\mathcal{F}_{i}$. Let $\leq$ be an ordering on $\mathcal{G}$ such that $(\mathcal{G}, \leq) \cong\left(\mathcal{F}_{i}, \leq_{i}\right)$. Then:

(1) $(\mathcal{G}, \leq) \models$ Same $(i)$ if and only if $\left(\varepsilon, \alpha_{i}\right)>1 \Longleftrightarrow\left(\varepsilon, \beta_{i}\right)>1$.

(2) $(\mathcal{G}, \leq) \models \operatorname{Different}(i)$ if and only if $\left(\varepsilon, \alpha_{i}\right)>1 \Longleftrightarrow\left(\varepsilon, \beta_{i}\right)<1$.

This lemma will be proved later. We will now show how to use Lemma 9 to complete proof.

Lemma 10. $\mathcal{G}$ has no computable presentation with a computable ordering.

Proof. Let $i$ be an index for $\left(\mathcal{F}_{i}, \leq_{i}\right)$ a computable presentation of $\mathcal{G}$ with a computable left-ordering. Let $\leq$ be an ordering on $\mathcal{G}$ such that $(\mathcal{G}, \leq) \cong\left(\mathcal{F}_{i}, \leq_{i}\right)$. Now by Lemma 9 either $(\mathcal{G}, \leq) \models \operatorname{Same}(i)$ or $(\mathcal{G}, \leq) \models \operatorname{Different}(i)$ (but not both). Suppose first that $(\mathcal{G}, \leq) \models \operatorname{Same}(i)$. So $\left(\mathcal{F}_{i}, \leq_{i}\right) \models \operatorname{Same}(i)$. By definition, $\psi(i)=1$, say $\psi_{\text {at } t}(i)=1$. Then, in $\mathcal{H} / \mathcal{R}, p_{i}^{t} \alpha_{i}=-q_{i}^{t} \beta_{i}$. So $\left(\varepsilon, \alpha_{i}\right)>1$ if and only if $\left(\varepsilon, \beta_{i}\right)<1$, contradicting Lemma 9 and the assumption that $(\mathcal{G}, \leq) \models \operatorname{Same}(i)$. The case of $(\mathcal{G}, \leq) \models \operatorname{Different}(i)$ is similar. Thus $\mathcal{G}$ has no computable copy with a computable left-ordering.

All that remains to prove Theorem 2 is to define $\operatorname{Same}(i)$ and $\operatorname{Different}(i)$ and to prove Lemma 9 .

\section{Same $(i)$, Different $(i)$, And the Proof of Lemma 9}

To define Same $(i)$, we would like to come up with an existential formula which says that $\left(\varepsilon, \alpha_{i}\right)>1 \Longleftrightarrow\left(\varepsilon, \beta_{i}\right)>1$. A first attempt might be to try to find an existential formula defining $\left(\varepsilon, \alpha_{i}\right)$ and an existential formula defining $\left(\varepsilon, \beta_{i}\right)$. This cannot be done, but it will be helpful to think about how we might try to do this.

We will consider the problem of recognizing $\alpha_{i}$ and $\beta_{i}$ inside of $\mathcal{H} / \mathcal{R}$ by their actions on $\mathcal{N}$. Note that $\alpha_{i}$ has the property that $\varphi_{\alpha_{i}}\left(v_{i, 0}\right)=v_{i, \alpha_{i}} \neq 0$, but $\varphi_{p_{i} \alpha_{i}}\left(v_{i, 0}\right)=v_{i, 0}$. So $\alpha_{i}$ acts with order $p_{i}$ on some element of $\mathcal{N}$. In fact, it is not hard to see that the only elements which act with order $p_{i}$ on an element of $\mathcal{N}$ are the multiples $n \alpha_{i}$ of $\alpha_{i}$ where $p_{i} \nmid n$. (Note that if $\alpha_{i}$ acts with order $p_{i}$ on a word in $\mathcal{N}$, then it either fixes or acts with order $p_{i}$ on each letter in that word, and it acts with order $p_{i}$ on at least one letter.)

One difficulty we have is that $\mathcal{H} / \mathcal{R}$ and $\mathcal{N}$ are not existentially definable inside of $\mathcal{G}$. The problem is that if some element of $\mathcal{G}$ satisfies a certain existential formula, then every conjugate of $\mathcal{G}$ does as well. So it is only possible to define subsets of $\mathcal{G}$ which are closed under conjugation. Given $S \subseteq \mathcal{G}$, let $S^{\mathcal{G}}$ be the set of all conjugates of $S$ by elements of $\mathcal{G}$. 
In this section, we will take for granted the following lemma about existential definability in $\mathcal{G}$. It will be proved in the following section. The lemma says that we can find $\mathcal{H} / \mathcal{R}$ inside of $\mathcal{G}$, up to conjugation, by an existential formula.

Lemma 11. $(\mathcal{H} / \mathcal{R})^{\mathcal{G}}$ is $\exists$-definable within $\mathcal{G}$ without parameters.

The different conjugates of $\mathcal{H} / \mathcal{R}$ cannot be distinguished from each other. Instead, we will try to always work inside a single conjugate of $\mathcal{H} / \mathcal{R}$. The following lemma tells us when we can do this.

Lemma 12. Suppose that $r, s \in(\mathcal{H} / \mathcal{R})^{\mathcal{G}}$ and $r s \in(\mathcal{H} / \mathcal{R})^{\mathcal{G}}$. Then there is $A \in \mathcal{N}$ and $g, h \in \mathcal{H} / \mathcal{R}$ such that

$$
r=(A, 0)(\varepsilon, g)\left(A^{-1}, 0\right)
$$

and

Thus $r$ and $s$ commute.

$$
s=(A, 0)(\varepsilon, h)\left(A^{-1}, 0\right) .
$$

The following remarks will be helpful not only here, but throughout the rest of the paper. They can all be checked by an easy computation.

Remark 13. If $r \in(\mathcal{H} / \mathcal{R})^{\mathcal{G}}$, then for some $A \in \mathcal{N}$ and $f \in \mathcal{H} / \mathcal{R}$ we can write $r$ in the form

$$
r=(A, 0)(\varepsilon, f)\left(A^{-1}, 0\right) .
$$

Remark 14. Let $r=(A, f)$ be an element of $(\mathcal{H} / \mathcal{R})^{\mathcal{G}}$. If $K \subseteq \mathcal{H} / \mathcal{R}$, then $r \in K^{\mathcal{G}}$ if and only if $f \in K$.

Remark 15. If $\varphi_{g}(B)=B$, then

$$
(A B, 0)(\varepsilon, g)(A B, 0)^{-1}=(A, 0)(\varepsilon, g)(A, 0)^{-1} .
$$

Proof of Lemma 12. Using Remark 13, let

$$
\begin{aligned}
r & =(A, 0)(\varepsilon, g)\left(A^{-1}, 0\right) \quad s=(B, 0)(\varepsilon, h)\left(B^{-1}, 0\right) \\
r s & =(C, 0)(\varepsilon, g+h)\left(C^{-1}, 0\right) .
\end{aligned}
$$

By conjugating $r$ and $s$ by some further element of $\mathcal{G}$ (and noting that the conclusion of the lemma is invariant under conjugation), we may assume that $A^{-1} B$ is a reduced word, that is, that $A$ and $B$ have no common non-trivial initial segment. Using Remark 15, we may assume that $A \varphi_{g}\left(A^{-1}\right), B \varphi_{h}\left(B^{-1}\right)$, and $C \varphi_{g+h}\left(C^{-1}\right)$ are reduced words. Indeed, if, for example, $A \varphi_{g}\left(A^{-1}\right)$ was not a reduced word, then we could write $A=A^{\prime} B$ where $B$ is a word which is fixed by $\varphi_{g}$, and such that $A^{\prime} \varphi_{g}\left(A^{\prime-1}\right)$ is a reduced word. Then, by Remark 15 ,

$$
(A, 0)(\varepsilon, g)(A, 0)^{-1}=\left(A^{\prime} B, 0\right)(\varepsilon, g)\left(A^{\prime} B, 0\right)^{-1}=\left(A^{\prime}, 0\right)(\varepsilon, g)\left(A^{\prime}, 0\right)^{-1} .
$$

So we may replace $A$ by $A^{\prime}$.

We have

$$
(A, 0)(\varepsilon, g)\left(A^{-1}, 0\right)(B, 0)(\varepsilon, h)\left(B^{-1}, 0\right)=(C, 0)(\varepsilon, g+h)\left(C^{-1}, 0\right) .
$$

Multiplying out the first coordinates, we get

$$
A \varphi_{g}\left(A^{-1}\right) \varphi_{g}(B) \varphi_{g+h}\left(B^{-1}\right)=C \varphi_{g+h}\left(C^{-1}\right)
$$

By the assumptions we made above, both sides are reduced words. $A$ is an initial segment of the left hand side, so it must be an initial segment of the right hand 
side, and hence an initial segment of $C$. On the other hand, taking inverses of both sides, we get

$$
\varphi_{g+h}(B) \varphi_{g}\left(B^{-1}\right) \varphi_{g}(A) A^{-1}=\varphi_{g+h}(C) C^{-1} .
$$

Once again both sides are reduced words, and $\varphi_{g+h}(B)$ is an initial segment of the left hand side, and hence of $\varphi_{g+h}(C)$. But then $B$ is an initial segment of $C$. So it must be that $A$ is an initial segment of $B$ or vice versa. This contradicts one of our initial assumptions unless $A$ or $B$ (or both) is the trivial word. Suppose it was $A$ (the case of $B$ is similar). Then

$$
\varphi_{g}(B) \varphi_{g+h}\left(B^{-1}\right)=C \varphi_{g+h}\left(C^{-1}\right)
$$

and both sides are reduced words. Then we get that $C=B$ and $C=\varphi_{g}(B)$. So

$$
r=(\varepsilon, g)=(B, 0)(\varepsilon, g)(B, 0)^{-1}
$$

by Remark 15 .

Above, we noted that the set $\left\{n \alpha_{i}: p_{i} \nmid n\right\}$ is the set of elements of $\mathcal{H} / \mathcal{R}$ which act with order $p_{i}$ on an element of $\mathcal{N}$. Our next goal is to show that if we close under conjugation, then this set (and a few other similar sets) are definable. The key is the following remark which follows easily from Lemma 12

Remark 16. Fix $r, s_{1}, s_{2} \in(\mathcal{H} / \mathcal{R})^{\mathcal{G}}$. Suppose that $r s_{1} \in(\mathcal{H} / \mathcal{R})^{\mathcal{G}}$ and $r s_{2} \in(\mathcal{H} / \mathcal{R})^{\mathcal{G}}$ but $s_{1}$ and $s_{2}$ do not commute. By Lemma 12 we can write

$$
\begin{aligned}
r & =(A, 0)(\varepsilon, f)\left(A^{-1}, 0\right)=(B, 0)(\varepsilon, f)\left(B^{-1}, 0\right) \\
s_{1} & =(A, 0)(\varepsilon, g)\left(A^{-1}, 0\right) \\
s_{2} & =(B, 0)(\varepsilon, h)\left(B^{-1}, 0\right) .
\end{aligned}
$$

Then there is some element of $\mathcal{N}$ which is fixed by $\varphi_{f}$ but which is not fixed by $\varphi_{g}$.

Indeed, since $(A, 0)(\varepsilon, f)\left(A^{-1}, 0\right)=(B, 0)(\varepsilon, f)\left(B^{-1}, 0\right)$, we see that

$$
B^{-1} A=\varphi_{f}\left(B^{-1} A\right) \text {. }
$$

Suppose for the sake of contradiction that $\varphi_{g}$ also fixes $B^{-1} A$. Then

$$
s_{1}=(A, 0)\left(A^{-1} B, 0\right)(\varepsilon, g)\left(B^{-1} A, 0\right)\left(A^{-1}, 0\right)=(B, 0)(\varepsilon, g)\left(B^{-1}, 0\right) .
$$

So $s_{1}$ and $s_{2}$ would commute. This is a contradiction. So there is some element of $\mathcal{N}$ which is fixed by $\varphi_{f}$ but which is not fixed by $\varphi_{g}$.

Lemma 17. There are $\exists$-formulas which express each of the following statements about an element $a$ in $\mathcal{G}$ :

(1) $a \in\left\{n \alpha_{i}: p_{i} \nmid n\right\}^{\mathcal{G}}$.

(2) $a \in\left\{n \beta_{i}: q_{i} \nmid n\right\}^{\mathcal{G}}$.

(3) $a \in\left\{n \gamma_{i}: r_{i} \nmid n\right\}^{\mathcal{G}}$.

(4) $a \in\left\{n\left(\alpha_{i}-\gamma_{i}\right): p_{i}, r_{i} \nmid n\right\}^{\mathcal{G}}$.

(5) $a \in\left\{n\left(\beta_{i}-\gamma_{i}\right): q_{i}, r_{i} \nmid n\right\}^{\mathcal{G}}$

Proof. For (1), we claim that $a \in\left\{n \alpha_{i}: p_{i} \nmid n\right\}^{\mathcal{G}}$ if and only if $a \in(\mathcal{H} / \mathcal{R})^{\mathcal{G}}$ and there is $b \in(\mathcal{H} / \mathcal{R})^{\mathcal{G}}$ such that $a^{p_{i}} b \in(\mathcal{H} / \mathcal{R})^{\mathcal{G}}$ but $a$ and $b$ do not commute. This is expressed by an $\exists$-formula by Lemma 11 .

Suppose that $a$ satisfies this $\exists$-formula, as witnessed by $b$. Let $a=(A, f)$ and $b=(B, g)$. Then by Remark [16 (taking $r=a^{p_{i}}, s_{1}=a$, and $\left.s_{2}=b\right)$, there is an element of $\mathcal{N}$ which is fixed by $\varphi_{p_{i} f}$ but not by $\varphi_{f}$. Thus we see that $p_{i} \bar{f}=0$ but $\bar{f} \neq 0$ in $\mathcal{H} / \mathcal{V}_{i}$, and $f=n \alpha_{i}$ for some $n$ with $p_{i} \nmid n$. (It must be in $\mathcal{H} / \mathcal{V}_{i}$, because 
this cannot happen in any of $\mathcal{H} / \mathcal{V}_{j}$ for $j \neq i$, or $\mathcal{H} / \mathcal{W}_{j}, \mathcal{H} / \mathcal{X}_{j}, \mathcal{H} / \mathcal{Y}_{j}$, or $\mathcal{H} / \mathcal{Z}_{j}$.) Thus by Remark 14 $a \in\left\{n \alpha_{i}: p_{i} \nmid n\right\}^{\mathcal{G}}$.

On the other hand, suppose that $a \in\left\{n \alpha_{i}: p_{i} \nmid n\right\}^{\mathcal{G}}$. Write

$$
a=(A, 0)\left(\varepsilon, n \alpha_{i}\right)\left(A^{-1}, 0\right) .
$$

with $p_{i}$ not dividing $n$. Then let $b=\left(A v_{i, 0}, 0\right)\left(\varepsilon, n \alpha_{i}\right)\left(\left(A v_{i, 0}\right)^{-1}, 0\right)$. By Remark 15. since $\varphi_{n p_{i} \alpha_{i}}\left(v_{i, 0}\right)=v_{i, 0}$, we have

$$
a^{p_{i}}=(A, 0)\left(\varepsilon, n p_{i} \alpha_{i}\right)\left(A^{-1}, 0\right)=\left(A v_{i, 0}, 0\right)\left(\varepsilon, n p_{i} \alpha_{i}\right)\left(\left(A v_{i, 0}\right)^{-1}, 0\right) .
$$

So $a^{p_{i}} b \in(\mathcal{H} / \mathcal{R})^{\mathcal{G}}$. On the other hand,

$$
a b=\left(A \varphi_{n \alpha_{i}}\left(v_{i, 0}\right) \varphi_{2 n \alpha_{i}}\left(v_{i, 0}\right)^{-1} \varphi_{2 n \alpha_{i}}\left(A^{-1}\right), 2 n \alpha_{i}\right)
$$

and

$$
b a=\left(A v_{i, 0} \varphi_{n \alpha_{i}}\left(v_{i, 0}\right)^{-1} \varphi_{2 n \alpha_{i}}\left(A^{-1}\right), 2 n \alpha_{i}\right) .
$$

So $a$ does not commute with $b$ since $\varphi_{n \alpha_{i}}\left(v_{i, 0}\right)=v_{i, n \alpha_{i}} \neq v_{i, 0}$. The proofs of (2) and (3) are similar.

For (4), we claim that $a \in\left\{n\left(\alpha_{i}-\gamma_{i}\right): p_{i}, r_{i} \nmid n\right\}^{\mathcal{G}}$ if and only if there are $b_{1} \in\left\{n \alpha_{i}: p_{i} \nmid n\right\}^{\mathcal{G}}, b_{2} \in\left\{n \gamma_{i}: r_{i} \nmid n\right\}^{\mathcal{G}}$, and $c \in(\mathcal{H} / \mathcal{R})^{\mathcal{G}}$ such that $a=b_{1} b_{2}^{-1}$, $a c, a b_{1} \in(\mathcal{H} / \mathcal{R})^{\mathcal{G}}$, and $c$ does not commute with $b_{1}$.

Suppose that there are such $b_{1}, b_{2}$, and $c$. We can write $b_{1}=\left(B_{1}, m \alpha_{i}\right)$ with $p_{i} \nmid$ $m$ and $b_{2}=\left(B_{2}, n \gamma_{i}\right)$ with $r_{i} \nmid \gamma_{i}$. Thus we can write $a=b_{1} b_{2}^{-1}=\left(A, m \alpha_{i}-n \gamma_{i}\right)$. By Remark 16 (with $r=a, s_{1}=b_{1}$, and $s_{2}=c$ ), $\varphi_{m \alpha_{i}-n \gamma_{i}}$ fixes some element of $\mathcal{N}$ which is not fixed by $\varphi_{m \alpha_{i}}$. Thus, in one of $\mathcal{H} / \mathcal{V}_{j}, \mathcal{H} / \mathcal{W}_{j}, \mathcal{H} / \mathcal{X}_{j}, \mathcal{H} / \mathcal{Y}_{j}$, or $\mathcal{H} / \mathcal{Z}_{j}$ for some $j$ we have $m \bar{\alpha}_{i}-n \bar{\gamma}_{i}=0$ but $m \bar{\alpha}_{i} \neq 0$. Since $p_{i} \nmid m$, it must be in $\mathcal{H} / \mathcal{Y}_{i}$. So $n=m$. Note that $p_{i}$ and $r_{i}$ do not divide $n$.

On the other hand, suppose that $a \in\left\{n\left(\alpha_{i}-\gamma_{i}\right): p_{i}, r_{i} \nmid n\right\}^{\mathcal{G}}$. Then write

$$
a=(A, 0)\left(\varepsilon, n \alpha_{i}-n \gamma_{i}\right)\left(A^{-1}, 0\right) .
$$

with $p_{i}$ and $r_{i}$ not dividing $n$. Let

$$
b_{1}=(A, 0)\left(\varepsilon, n \alpha_{i}\right)\left(A^{-1}, 0\right) \text { and } b_{2}=(A, 0)\left(\varepsilon, n \gamma_{i}\right)\left(A^{-1}, 0\right)
$$

and let

$$
c=\left(A y_{i, 0}, 0\right)\left(\varepsilon, n \alpha_{i}\right)\left(\left(A y_{i, 0}\right)^{-1}, 0\right) .
$$

Then $a=b_{1} b_{2}^{-1}$. Clearly $a b_{1} \in(\mathcal{H} / \mathcal{R})^{\mathcal{G}}$. Also, since $\varphi_{n \alpha_{i}-n \gamma_{i}}\left(y_{i, 0}\right)=y_{i, 0}$,

$$
a c=c a=\left(A y_{i, 0}, 0\right)\left(\varepsilon, 2 n \alpha_{i}-n \gamma_{i}\right)\left(\left(A y_{i, 0}\right)^{-1}, 0\right) .
$$

So $a c \in(\mathcal{H} / \mathcal{R})^{\mathcal{G}}$ and $a$ and $c$ commute. On the other hand, $b_{1}$ does not commute with $c$ since $\varphi_{\ell \alpha_{i}}\left(y_{i, 0}\right)=y_{i, \ell \alpha_{i}} \neq y_{i, 0}$ as $p_{i}$ does not divide $\ell$.

We will now define Same $(i)$ and $\operatorname{Different}(i)$.

Definition 18. Same $(i)$ says that there are $a, b$, and $c$ such that:

(1) $a, b, c$, and $a b$ are in $(\mathcal{H} / \mathcal{R})^{\mathcal{G}}$,

(2) $a>1 \Longleftrightarrow b>1$

(3) $a \in\left\{n \alpha_{i}: p_{i} \nmid n\right\}^{\mathcal{G}}$,

(4) $b \in\left\{n \beta_{i}: q_{i} \nmid n\right\}^{\mathcal{G}}$,

(5) $c \in\left\{n \gamma_{i}: r_{i} \nmid n\right\}^{\mathcal{G}}$,

(6) $a c^{-1} \in\left\{n\left(\alpha_{i}-\gamma_{i}\right): p_{i}, r_{i} \nmid n\right\}^{\mathcal{G}}$.

(7) $b c^{-1} \in\left\{n\left(\beta_{i}-\gamma_{i}\right): q_{i}, r_{i} \nmid n\right\}^{\mathcal{G}}$. 
$\operatorname{Different}(i)$ is defined in the same way as $\operatorname{Same}(i)$, except that in (2) we ask that $a>1$ if and only if $b<1$.

Suppose, for simplicity, that $a, b$, and $c$ are all in $\mathcal{H} / \mathcal{R}$. Then we would have that $a=\left(\varepsilon, \ell \alpha_{i}\right), b=\left(\varepsilon, m \beta_{i}\right)$, and $c=\left(\varepsilon, n \gamma_{i}\right)$. Now $a c^{-1}=\left(\varepsilon, \ell \alpha_{i}-n \gamma_{i}\right)$ is a power of $\left(\varepsilon, \alpha_{i}-\gamma_{i}\right)$, and so $\ell=n$. Similarly, $b c^{-1}=\left(\varepsilon, m \beta_{i}-n \gamma_{i}\right)$ is a power of $\left(\varepsilon, \beta_{i}-\gamma_{i}\right)$, and so $m=n$. Thus $\ell=m$. Since $\left(\varepsilon, \ell \alpha_{i}\right)>1 \Longleftrightarrow\left(\varepsilon, \ell \beta_{i}\right)>1$, $\left(\varepsilon, \alpha_{i}\right)>1 \Longleftrightarrow\left(\varepsilon, \beta_{i}\right)>1$. Checking that this works even if $a, b$, and $c$ are conjugates of $\mathcal{H} / \mathcal{R}$ is the heart of Lemma 19

Lemma 19. Let $\leq$ be a left-ordering on $\mathcal{G}$. Then:

(1) If $\left(\varepsilon, \alpha_{i}\right)>1 \Longleftrightarrow\left(\varepsilon, \beta_{i}\right)>1$, then $(\mathcal{G}, \leq) \models \operatorname{Same}(i)$.

(2) If $\left(\varepsilon, \alpha_{i}\right)>1 \Longleftrightarrow\left(\varepsilon, \beta_{i}\right)<1$, then $(\mathcal{G}, \leq) \models \operatorname{Different}(i)$.

(3) If $\psi(i) \downarrow$, then $\left(\varepsilon, \alpha_{i}\right)>1 \Longleftrightarrow\left(\varepsilon, \beta_{i}\right)>1$ if and only if $(\mathcal{G}, \leq) \models \operatorname{Same}(i)$.

(4) If $\psi(i) \downarrow$, then $\left(\varepsilon, \alpha_{i}\right)>1 \Longleftrightarrow\left(\varepsilon, \beta_{i}\right)<1$ if and only if $(\mathcal{G}, \leq) \models \operatorname{Different}(i)$.

Proof. First, for $(1)$, suppose that $\left(\varepsilon, \alpha_{i}\right)>1 \Longleftrightarrow\left(\varepsilon, \beta_{i}\right)>1$. Then $(\mathcal{G}, \leq) \models$ Same $(i)$ as witnessed by $c=\left(\varepsilon, \alpha_{i}\right), c=\left(\varepsilon, \beta_{i}\right)$, and $c=\left(\varepsilon, \gamma_{i}\right)$. (2) is similar.

Now for (3), suppose that $(\mathcal{G}, \leq) \models \operatorname{Same}(i)$ as witnessed by $a, b$, and $c$, and that $\psi(i) \downarrow$. Let $f, g$, and $h$ be the second coordinates of $a, b$, and $c$ respectively. Write $f=\ell \alpha_{i}$ with $p_{i} \nmid \ell, g=m \beta_{i}$ with $q_{i} \nmid m$, and $h=n \gamma_{i}$ with $r_{i} \nmid h$. Then since $f-h$ is a multiple of $\alpha_{i}-\gamma_{i}, \ell=n$. Similarly, $m=n$, and so $\ell=m$.

Since $a b \in(\mathcal{H} / \mathcal{R})^{\mathcal{G}}$ and $a$ and $b$ commute, by Lemma 12 we can write

$$
a=(B, 0)\left(\varepsilon, \ell \alpha_{i}\right)(B, 0)^{-1}
$$

and

$$
b=(B, 0)\left(\varepsilon, \ell \beta_{i}\right)(B, 0)^{-1} .
$$

Now since $\psi(i) \downarrow$, in $\mathcal{H} / \mathcal{R}$ either $p_{i}^{t} \alpha_{i}=q_{i}^{t} \beta_{i}$ or $p_{i}^{t} \alpha_{i}=-q_{i}^{t} \beta_{i}$ for some $t$. In the second case, $a^{p_{i}^{t}}=b^{-q_{i}^{t}}$ which contradicts the fact that $a>1 \Longleftrightarrow b>1$. Thus $p_{i}^{t} \alpha_{i}=q_{i}^{t} \beta_{i}$, and so $\left(\varepsilon, \alpha_{i}\right)>1 \Longleftrightarrow\left(\varepsilon, \beta_{i}\right)>1$.

(4) is proved similarly.

Proof of Lemma 9. We will prove $(1):(\mathcal{G}, \leq) \models \operatorname{Same}(i)$ if and only if $\left(\varepsilon, \alpha_{i}\right)>$ $1 \Longleftrightarrow\left(\varepsilon, \beta_{i}\right)>1$. The proof of $(2)$ is similar. The right to left direction follows immediately from (1) of Lemma 19, For the left to right direction, suppose that $\left(\mathcal{F}_{i}, \leq_{i}\right) \models$ Same $(i)$. Then $\psi(i) \downarrow$. Then the lemma follows from (3) of Lemma 19.

\section{An Existential Definition of $(\mathcal{H} / \mathcal{R})^{\mathcal{G}}$}

The goal of this section is to prove Lemma 11, which says that $(\mathcal{H} / \mathcal{R})^{\mathcal{G}}$ is definable within $\mathcal{G}$ by an existential formula. To prove this lemma, we will first have to give a detailed analysis of which elements of $\mathcal{G}$ commute with each other.

The first lemma is the analogue of the following well-known fact about free groups: two elements $a$ and $b$ in a free group commute if and only if there is $c$ such that $a=c^{m}$ and $b=c^{n}$ (see [LS01, Proposition 2.17]).

Lemma 20. Let $r, s \in \mathcal{G}$ commute. Then there are $W, V \in \mathcal{N}, x, y, z \in \mathcal{H} / \mathcal{R}$, and $k, \ell \in \mathbb{Z}$ such that

and

$$
r=(W, 0)(V, x)^{k}(\varepsilon, y)(W, 0)^{-1}
$$

$$
s=(W, 0)(V, x)^{\ell}(\varepsilon, z)(W, 0)^{-1} .
$$


If $k \neq 0$ then $\varphi_{z}(V)=V$, and if $\ell \neq 0$ then $\varphi_{y}(V)=V$.

It is easy to check that two such elements commute.

Proof. Suppose that $r s=s r$. Let $r=(A, g)$ and $s=(B, h)$. Then we find that

$$
\begin{aligned}
r s & =(A, g)(B, h) \\
& =\left(A \varphi_{g}(B), g+h\right) \\
s r & =(B, h)(A, g) \\
& =\left(B \varphi_{h}(A), g+h\right) .
\end{aligned}
$$

So $A \varphi_{g}(B)=B \varphi_{h}(A)$ in $\mathcal{N}$. Write

$$
A=a_{0} \cdots a_{m-1} \text { and } B=b_{0} \cdots b_{n-1}
$$

as reduced words. So

$$
a_{0} \cdots a_{m-1} \varphi_{g}\left(b_{0}\right) \cdots \varphi_{g}\left(b_{n-1}\right)=b_{0} \cdots b_{n-1} \varphi_{h}\left(a_{0}\right) \cdots \varphi_{h}\left(a_{m-1}\right) .
$$

We divide into several cases.

Case 1. $A$ is the trivial word.

We must have $B=\varphi_{g}(B)$. Then $r=(\varepsilon, g)$ and $s=(B, h)$. Take $W=\varepsilon, V=B$, $x=h, y=g, z=0, k=0$, and $\ell=1$.

Case 2. $B$ is the trivial word.

We must have $A=\varphi_{h}(A)$. Then $r=(A, g)$ and $s=(\varepsilon, h)$. Take $W=\varepsilon, V=A$, $x=g, y=0, z=h, k=1$, and $\ell=0$.

Case 3. Neither $A$ nor $B$ is the trivial word, and both $A \varphi_{g}(B)$ and $B \varphi_{h}(A)$ are reduced words.

We have $A \varphi_{g}(B)=B \varphi_{h}(A)$ as reduced words. Assume without loss of generality that $|A|=m \geq n=|B|$. Then $n, m>0$ and

$$
a_{0} \cdots a_{m-1} \varphi_{g}\left(b_{0}\right) \cdots \varphi_{g}\left(b_{n-1}\right)=b_{0} \cdots b_{n-1} \varphi_{h}\left(a_{0}\right) \cdots \varphi_{h}\left(a_{m-1}\right)
$$

as reduced words. So

$$
\begin{aligned}
a_{i} & =b_{i} & & \text { for } 0 \leq i<n \\
a_{i} & =\varphi_{h}\left(a_{i-n}\right) & & \text { for } n \leq i<m \\
\varphi_{g}\left(b_{i}\right) & =\varphi_{h}\left(a_{m-n+i}\right) & & \text { for } 0 \leq i<n .
\end{aligned}
$$

Let $d=\operatorname{gcd}(m, n)$. (This is where we use the fact that $m, n>0$.) Let $n^{\prime}=n / d$ and $m^{\prime}=m / d$.

Given $p, q \geq 0$, write $i=q n-p m+r$ with $0 \leq r<d$ and assume that $0 \leq i<m$. Note that every $i, 0 \leq i<m$, can be written in such a way. We claim that

$$
a_{i}=\varphi_{q h-p g}\left(a_{r}\right) .
$$

We argue by induction, ordering pairs $(q, p)$ lexicographically. For the base case $p=q=0$ we note that $a_{r}=\varphi_{0}\left(a_{r}\right)$. Otherwise, if $n \leq i<m$, then we must have $q>0$. By the induction hypothesis, $a_{i-n}=\varphi_{(q-1) h-p g}\left(a_{r}\right)$. So

$$
a_{i}=\varphi_{h}\left(a_{i-n}\right)=\varphi_{q h-p g}\left(a_{r}\right) .
$$


If $0 \leq i<n$, and $(q, p) \neq(0,0)$, then $q>0$ and $p>0$. Note that $a_{m-n+i}=$ $\varphi_{(q-1) h-(p-1) g}\left(a_{r}\right)$ by the induction hypothesis and so

$$
a_{i}=b_{i}=\varphi_{h-g}\left(a_{m-n+i}\right)=\varphi_{q h-p g}\left(a_{r}\right) .
$$

This completes the induction.

Write $d=q n-p m$ with $p, q \geq 0$. Let $f=q h-p g$. Then each $i, 0 \leq i<m$, can be written as $i=k d+r$ with $0 \leq r<d$, and so $a_{i}=\varphi_{k f}\left(a_{r}\right)$.

Let $C=a_{0} \cdots a_{d-1}$. Then

$$
A=C \varphi_{f}(C) \cdots \varphi_{\left(m^{\prime}-1\right) f}(C)
$$

and so

$$
r=(A, g)=(C, f)^{m^{\prime}}\left(\varepsilon, g-m^{\prime} f\right) .
$$

Since for $0 \leq i<n, a_{i}=b_{i}$, we have

$$
s=(B, h)=(C, f)^{n^{\prime}}\left(\varepsilon, h-n^{\prime} f\right) .
$$

This is in the desired form: take $W=\varepsilon, V=C, x=f, y=g-m^{\prime} f, z=h-n^{\prime} f$, $k=m^{\prime}$, and $\ell=n^{\prime}$.

We still have to show that $\varphi_{y}(V)=\varphi_{z}(V)=V$. Noting that

$$
\left(n^{\prime} q-1\right) n-\left(n^{\prime} p\right) m=n^{\prime}(q n-p m)-n=n^{\prime} d-n=0
$$

we have, for all $0 \leq r<d$,

$$
a_{r}=\varphi_{\left(n^{\prime} q-1\right) h-n^{\prime} p g}\left(a_{r}\right)=\varphi_{n^{\prime} f-h}\left(a_{r}\right) .
$$

Similarly,

$$
a_{r}=\varphi_{m^{\prime} f-g}\left(a_{r}\right) .
$$

Hence $\varphi_{g-m^{\prime} f}(C)=\varphi_{h-n^{\prime} f}(C)=C$.

Case 4. Neither $A$ nor $B$ is the trivial word, and both $B^{-1} A$ and $\varphi_{h}(A) \varphi_{g}(B)^{-1}$ are reduced words.

Note that $B^{-1} A=\varphi_{h}(A) \varphi_{g}(B)^{-1}$. We can make a transformation to reduce this to the previous case. Let

$$
A^{\prime}=B^{-1} \quad B^{\prime}=\varphi_{h}(A) \quad g^{\prime}=-h \quad h^{\prime}=g .
$$

Then $A^{\prime} \varphi_{g^{\prime}}\left(B^{\prime}\right)=B^{\prime} \varphi_{h^{\prime}}\left(A^{\prime}\right)$ and these are reduced words. Hence by the previous case there are $C \in \mathcal{N}, f \in \mathcal{H} / \mathcal{R}$, and $m, n \in \mathbb{Z}$ such that

$$
\left(A^{\prime}, g^{\prime}\right)=(C, f)^{m}\left(\varepsilon, g^{\prime}-m f\right)
$$

and

$$
\left(B^{\prime}, h^{\prime}\right)=(C, f)^{n}\left(\varepsilon, h^{\prime}-n f\right)
$$

and such that $\varphi_{g^{\prime}-m f}(C)=C$ and $\varphi_{h^{\prime}-n f}(C)=C$. Now

$$
\begin{aligned}
(A, g) & =(\varepsilon,-h)\left(\varphi_{h}(A), g\right)(\varepsilon, h) \\
& =(\varepsilon,-h)\left(B^{\prime}, h^{\prime}\right)(\varepsilon, h) \\
& =(\varepsilon,-h)(C, f)^{n}\left(\varepsilon, h^{\prime}-n f\right)(\varepsilon, h) \\
& =\left(\varphi_{-h}(C), f\right)^{n}(\varepsilon, g-n f) .
\end{aligned}
$$


Note that $\varphi_{g-n f}(C)=\varphi_{h^{\prime}-n f}(C)=C$, and so $\varphi_{g-n f}\left(\varphi_{-h}(C)\right)=\varphi_{-h}(C)$. Similarly,

$$
\begin{aligned}
(B, h) & =(\varepsilon,-h)\left(B^{-1},-h\right)^{-1}(\varepsilon, h) \\
& =(\varepsilon,-h)\left(A^{\prime}, g^{\prime}\right)^{-1}(\varepsilon, h) \\
& =(\varepsilon,-h)\left(\varepsilon, g^{\prime}-m f\right)^{-1}(C, f)^{-m}(\varepsilon, h) \\
& =(\varepsilon, m f)(C, f)^{-m}(\varepsilon, h) \\
& =\left(\varphi_{m f}(C), f\right)^{-m}(\varepsilon, h+m f) .
\end{aligned}
$$

Since $\varphi_{h+m f}(C)=\varphi_{g^{\prime}-m f}(C)=C, \varphi_{m f}(C)=\varphi_{-h}(C)$. So

$$
(B, h)=\left(\varphi_{-h}(C), f\right)^{-m}(\varepsilon, h+m f) .
$$

This completes this case, taking $W=\varepsilon, V=\varphi_{-h}(C), x=f, y=g-n f$, $z=h+m f, k=n$, and $\ell=-m$.

Case 5. $|A|=1, B$ is not the trivial word, and neither $A \varphi_{g}(B)=B \varphi_{h}(A)$ nor $B^{-1} A=\varphi_{h}(A) \varphi_{g}\left(B^{-1}\right)$ are reduced words.

Let $A=a$. Then $a^{-1}=\varphi_{g}\left(b_{0}\right)$ and $b_{n-1}=\varphi_{h}\left(a^{-1}\right)$. Recall that $B=b_{0} \cdots b_{n-1}$. From the non-reduced words $A \varphi_{g}(B)=B \varphi_{h}(A)$, we get, as reduced words,

$$
\varphi_{g}\left(b_{1}\right) \varphi_{g}\left(b_{2}\right) \cdots \varphi_{g}\left(b_{n-1}\right)=b_{0} b_{1} \cdots b_{n-2} .
$$

Then, for $0 \leq i<n-1$ we get $\varphi_{g}\left(b_{i+1}\right)=b_{i}$. Thus $a=\varphi_{n g+h}(a)$. Also, letting $C=b_{0}$,

$$
r=\left(\varphi_{g}(C)^{-1}, g\right)=(C,-g)^{-1}
$$

and

$$
s=(C,-g)^{n}(\varepsilon, h+n g)
$$

Note that $\varphi_{h+n g}(C)=\varphi_{h+n g}\left(b_{0}\right)=b_{0}$ since $a=\varphi_{n g+h}(a)$ and $b_{0}=\varphi_{-g}\left(a^{-1}\right)$.

So in this case we take $W=\varepsilon, V=C, x=g, y=0, z=h+n g, k=-1$, and $\ell=n$.

Case 6. $|B|=1, A$ is not the trivial word, and neither $A \varphi_{g}(B)=B \varphi_{h}(A)$ nor $B^{-1} A=\varphi_{h}(A) \varphi_{g}\left(B^{-1}\right)$ are reduced words.

This case is similar to the previous case.

Case 7. $|A|,|B| \geq 2$ and neither $A \varphi_{g}(B)=B \varphi_{h}(A)$ nor $B^{-1} A=\varphi_{h}(A) \varphi_{g}\left(B^{-1}\right)$ are reduced words.

We have $b_{n-1}=\varphi_{h}\left(a_{0}\right)^{-1}$ and $\varphi_{h}\left(a_{m-1}\right)=\varphi_{g}\left(b_{n-1}\right)$ and so

$$
\varphi_{g}\left(a_{0}\right)=\varphi_{g}\left(a_{0}^{-1}\right)^{-1}=\varphi_{g-h}\left(b_{n-1}\right)^{-1}=a_{m-1}^{-1} .
$$

Letting

$$
A^{\prime}=a_{1} \cdots a_{m-2}=a_{0}^{-1} A \varphi_{g}\left(a_{0}\right)
$$

and

$$
B^{\prime}=a_{0}^{-1} b_{0} b_{1} \cdots b_{n-2}=a_{0}^{-1} B \varphi_{h}\left(a_{0}\right)
$$


we have

$$
\begin{aligned}
B^{\prime} \varphi_{h}\left(A^{\prime}\right) \varphi_{g}\left(B^{\prime}\right)^{-1} & =B^{\prime} b_{n-1} \varphi_{h}\left(a_{0}\right) \varphi_{h}\left(A^{\prime}\right) \varphi_{h}\left(a_{m-1}\right) \varphi_{g}\left(b_{n-1}\right)^{-1} \varphi_{g}\left(B^{\prime}\right)^{-1} \\
& =a_{0}^{-1} B \varphi_{h}(A) \varphi_{g}(B)^{-1} a_{m-1}^{-1} \\
& =a_{0}^{-1} A a_{m-1}^{-1} \\
& =A^{\prime}
\end{aligned}
$$

So $\left(A^{\prime}, g\right)$ and $\left(B^{\prime}, h\right)$ still commute.

Note that $\left|A^{\prime}\right|<|A|$ and $\left|B^{\prime}\right| \leq|B|$. So we only have to repeat this finitely many times until we are in one of the other cases. Thus, for some word $D$ we get reduced words

and

$$
A^{\prime}=D A \varphi_{g}\left(D^{-1}\right)
$$

$$
B^{\prime}=D B \varphi_{h}\left(D^{-1}\right)
$$

which fall into one of the other cases. So

$$
\left(A^{\prime}, g\right)=(C, f)^{m}(\varepsilon, g-m f)
$$

and

$$
\left(B^{\prime}, h\right)=(C, f)^{n}(\varepsilon, h-n f) .
$$

Thus

$$
r=\left(D A^{\prime} \varphi_{g}\left(D^{-1}\right), g\right)=(D, 0)\left(A^{\prime}, g\right)\left(D^{-1}, 0\right)
$$

and

$$
s=\left(D B^{\prime} \varphi_{h}\left(D^{-1}\right), h\right)=(D, 0)\left(B^{\prime}, h\right)\left(D^{-1}, 0\right)
$$

are in the desired form.

The next lemma gives a criterion for knowing that an element $r$ is in $(\mathcal{H} / \mathcal{R})^{\mathcal{G}}$, but it requires knowing that two particular elements $s_{1}$ and $s_{2}$ are not in $(\mathcal{H} / \mathcal{R})^{\mathcal{G}}$. This does not seem useful yet, but in Lemma 23 we will show that any three elements $s_{1}, s_{2}$, and $s_{3}$, such that $r$ commutes with each of them but $s_{1}, s_{2}$, and $s_{3}$ pairwise do not commute, give rise to two such elements which are not in $(\mathcal{H} / \mathcal{R})^{\mathcal{G}}$.

Lemma 21. Let $r, s_{1}, s_{2} \in \mathcal{G}$. Suppose that $r$ commutes with $s_{1}$ and $s_{2}$, but $s_{1}$ and $s_{2}$ do not commute. If $s_{1}, s_{2} \notin(\mathcal{H} / \mathcal{R})^{\mathcal{G}}$, then $r \in(\mathcal{H} / \mathcal{R})^{\mathcal{G}}$.

Proof. Suppose to the contrary that $r \notin(\mathcal{H} / \mathcal{R})^{\mathcal{G}}$. Since $r$ and $s_{1}$ commute, and $r$ and $s_{2}$ commute, by Lemma 20 we can write

$$
\begin{aligned}
r & =(A, 0)\left(C, f_{1}\right)^{m_{1}}\left(\varepsilon, g_{1}\right)\left(A^{-1}, 0\right)=(B, 0)\left(D, f_{2}\right)^{m_{2}}\left(\varepsilon, g_{2}\right)\left(B^{-1}, 0\right) \\
s_{1} & =(A, 0)\left(C, f_{1}\right)^{n_{1}}\left(\varepsilon, h_{1}\right)\left(A^{-1}, 0\right) \\
s_{2} & =(B, 0)\left(D, f_{2}\right)^{n_{2}}\left(\varepsilon, h_{2}\right)\left(B^{-1}, 0\right)
\end{aligned}
$$

Since $r, s_{1}$, and $s_{2}$ are not in $(\mathcal{H} / \mathcal{R})^{\mathcal{G}}, C$ and $D$ are non-trivial and $m_{1}, m_{2}, n_{1}, n_{2} \neq$ 0 . So $\varphi_{g_{1}}(C)=\varphi_{h_{1}}(C)=C$ and $\varphi_{g_{2}}(D)=\varphi_{h_{2}}(D)=D$. Moreover, we will argue that we may assume that

$$
C \varphi_{f_{1}}(C) \cdots \varphi_{\left(m_{1}-1\right) f_{1}}(C) \text { and } D \varphi_{f_{2}}(D) \cdots \varphi_{\left(m_{2}-1\right) f_{2}}(D)
$$

are reduced words. If the former is not a reduced word, then it must have length at least 2 , and we can write $C=a C^{\prime} \varphi_{f_{1}}\left(a^{-1}\right)$. Then

$$
C \varphi_{f_{1}}(C) \cdots \varphi_{\left(m_{1}-1\right) f_{1}}(C)=a C^{\prime} \varphi_{f_{1}}\left(C^{\prime}\right) \cdots \varphi_{\left(m_{1}-1\right) f_{1}}\left(C^{\prime}\right) \varphi_{m_{1} f_{1}}\left(a^{-1}\right)
$$


and so, since $\varphi_{g_{1}}$ fixes $C$ and hence $a$,

$$
r=(A a, 0)\left(C^{\prime}, f_{1}\right)^{m_{1}}\left(\varepsilon, g_{1}\right)\left(a^{-1} A^{-1}, 0\right) .
$$

Similarly,

$$
s_{1}=(A a, 0)\left(C^{\prime}, f_{1}\right)^{n_{1}}\left(\varepsilon, h_{1}\right)\left(a^{-1} A^{-1}, 0\right) .
$$

So we may replace $A$ by $A a$ and $C$ by $C^{\prime}$. We can continue to do this until $C \varphi_{f_{1}}(C) \cdots \varphi_{\left(m_{1}-1\right) f_{1}}(C)$ is a reduced word. The same argument works for $D \varphi_{f_{2}}(D) \cdots \varphi_{\left(m_{2}-1\right) f_{2}}(D)$.

Rearranging the two expressions for $r$, we get

$$
\left(B^{-1} A, 0\right)\left(C, f_{1}\right)^{m_{1}}\left(\varphi_{g_{1}}\left(A^{-1} B\right), g_{1}\right)=\left(D, f_{2}\right)^{m_{2}}\left(\varepsilon, g_{2}\right) .
$$

Looking at the first coordinate,

$$
\begin{aligned}
& B^{-1} A C \varphi_{f_{1}}(C) \varphi_{2 f_{1}}(C) \cdots \varphi_{\left(m_{1}-1\right) f_{1}}(C) \varphi_{m_{1} f_{1}+g_{1}}\left(A^{-1} B\right) \\
= & D \varphi_{f_{2}}(D) \varphi_{2 f_{2}}(D) \cdots \varphi_{\left(m_{2}-1\right) f_{2}}(D) .
\end{aligned}
$$

We claim that we can write $B^{-1} A=E_{2}^{-1} E_{1}$ where $\varphi_{g_{1}}\left(E_{1}\right)=\varphi_{h_{1}}\left(E_{1}\right)=E_{1}$ and $\varphi_{g_{2}}\left(E_{2}\right)=\varphi_{h_{2}}\left(E_{2}\right)=E_{2}$. Recall that

$$
C \varphi_{f_{1}}(C) \varphi_{2 f_{1}}(C) \cdots \varphi_{\left(m_{1}-1\right) f_{1}}(C)
$$

is a non-trivial reduced word. Taking a high enough power $\ell$, the length of

$$
\left(C \varphi_{f_{1}}(C) \varphi_{2 f_{1}}(C) \cdots \varphi_{\left(m_{1}-1\right) f_{1}}(C)\right)^{\ell}
$$

as a reduced word is more than twice the length of $B^{-1} A$. Then

$$
\begin{aligned}
& B^{-1} A\left(C \varphi_{f_{1}}(C) \varphi_{2 f_{1}}(C) \cdots \varphi_{\left(m_{1}-1\right) f_{1}}(C)\right)^{\ell} \varphi_{m_{1} f_{1}+g_{1}}\left(A^{-1} B\right) \\
= & \left(D \varphi_{f_{2}}(D) \varphi_{2 f_{2}}(D) \cdots \varphi_{\left(m_{2}-1\right) f_{2}}(D)\right)^{\ell} .
\end{aligned}
$$

We can write $B^{-1} A=E_{2}^{-1} E_{1}$ as a reduced word where $E_{2}^{-1}$ appears at the start of the right hand side when it is written as a reduced word, and $E_{1}$ cancels with the beginning of $\left(C \varphi_{f_{1}}(C) \varphi_{2 f_{1}}(C) \cdots \varphi_{\left(m_{1}-1\right) f_{1}}(C)\right)^{\ell}$. Thus $E_{1}$ is fixed by $\varphi_{g_{1}}$ and $\varphi_{h_{1}}$ since they fix each letter appearing in the word $\left(C \varphi_{f_{1}}(C) \varphi_{2 f_{1}}(C) \cdots \varphi_{\left(m_{1}-1\right) f_{1}}(C)\right)^{\ell}$, and $E_{2}$ is fixed by $\varphi_{g_{2}}$ and $\varphi_{h_{2}}$ since they fix each letter appearing in the right hand side.

Since $E_{2} B^{-1}=E_{1} A^{-1}$,

$$
\begin{aligned}
E_{2} B^{-1} r B E_{2}^{-1} & =\left(E_{1}, 0\right)\left(C, f_{1}\right)^{m_{1}}\left(\varepsilon, g_{1}\right)\left(E_{1}^{-1}, 0\right) \\
& =\left(E_{2}, 0\right)\left(D, f_{2}\right)^{m_{2}}\left(\varepsilon, g_{2}\right)\left(E_{2}^{-1}, 0\right) \\
E_{2} B^{-1} s_{1} B E_{2}^{-1} & =\left(E_{1}, 0\right)\left(C, f_{1}\right)^{n_{1}}\left(\varepsilon, h_{1}\right)\left(E_{1}^{-1}, 0\right) \\
E_{2} B^{-1} s_{2} B E_{2}^{-1} & =\left(E_{2}, 0\right)\left(D, f_{2}\right)^{n_{2}}\left(\varepsilon, h_{2}\right)\left(E_{2}^{-1}, 0\right) .
\end{aligned}
$$

So, applying the automorphism of $\mathcal{G}$ given by conjugating by $E_{2} B^{-1}$ (and noting that this automorphism fixes $(\mathcal{H} / \mathcal{R})^{\mathcal{G}}$ ) we may assume from the beginning that $\varphi_{g_{1}}(A)=\varphi_{h_{1}}(A)=A$ and $\varphi_{g_{2}}(B)=\varphi_{h_{2}}(B)=B$. Thus

$$
\begin{aligned}
r & =(A, 0)\left(C, f_{1}\right)^{m_{1}}\left(A^{-1}, 0\right)\left(\varepsilon, g_{1}\right)=(B, 0)\left(D, f_{2}\right)^{m_{2}}\left(B^{-1}, 0\right)\left(\varepsilon, g_{2}\right) \\
s_{1} & =(A, 0)\left(C, f_{1}\right)^{n_{1}}\left(A^{-1}, 0\right)\left(\varepsilon, h_{1}\right) \\
s_{2} & =(B, 0)\left(D, f_{2}\right)^{n_{2}}\left(B^{-1}, 0\right)\left(\varepsilon, h_{2}\right) .
\end{aligned}
$$


Now looking at the first coordinate, we have

$$
\begin{aligned}
& A C \varphi_{f_{1}}(C) \varphi_{2 f_{1}}(C) \cdots \varphi_{\left(m_{1}-1\right) f_{1}}(C) \varphi_{m_{1} f_{1}}(A)^{-1} \\
= & B D \varphi_{f_{2}}(D) \varphi_{2 f_{2}}(D) \cdots \varphi_{\left(m_{2}-1\right) f_{2}}(D) \varphi_{m_{2} f_{2}}(B)^{-1} .
\end{aligned}
$$

Our next step is to argue that we may assume that these are reduced words. Suppose that there was some cancellation, say $A=A^{\prime} a$ and $C=a^{-1} C^{\prime}$. Let $C^{*}=C^{\prime} \varphi_{f_{1}}\left(a^{-1}\right)$. Then

$$
\begin{aligned}
& A C \varphi_{f_{1}}(C) \varphi_{2 f_{1}}(C) \cdots \varphi_{\left(m_{1}-1\right) f_{1}}(C) \varphi_{m_{1} f_{1}}(A)^{-1} \\
= & A^{\prime} C^{*} \varphi_{f_{1}}\left(C^{*}\right) \varphi_{2 f_{1}}\left(C^{*}\right) \cdots \varphi_{\left(m_{2}-1\right) f_{1}}\left(C^{*}\right) \varphi_{m_{1} f_{1}}\left(A^{\prime}\right)^{-1} .
\end{aligned}
$$

Thus

$$
\begin{aligned}
r & =\left(A^{\prime}, 0\right)\left(C^{*}, f_{1}\right)^{m_{1}}\left(\varepsilon, g_{1}\right)\left(A^{\prime}, 0\right)^{-1} \\
s_{1} & =\left(A^{\prime}, 0\right)\left(C^{*}, f_{1}\right)^{n_{1}}\left(\varepsilon, h_{1}\right)\left(A^{\prime}, 0\right)^{-1} .
\end{aligned}
$$

Note that

$$
\left(C^{*}, f_{1}\right)^{m_{1}}=C^{*} \varphi_{f_{1}}\left(C^{*}\right) \varphi_{2 f_{1}}\left(C^{*}\right) \cdots \varphi_{\left(m_{1}-1\right) f_{1}}\left(C^{*}\right)
$$

is still a reduced word. If it was not a reduced word, then we would have $m_{1}>0$, $\left|C^{*}\right|>1$, and $\varphi_{f_{1}}\left(a^{-1}\right)=\varphi_{f_{1}}\left(a^{\prime}\right)^{-1}$, where $a^{\prime}$ is the first letter of $C^{*}$. Thus $a^{\prime}=a$ is the second letter of $C$, which together with the fact that the first letter of $C$ is $a^{-1}$ contradicts our assumption that $C$ is a reduced word. We have reduced the size of $A$, so after finitely many reductions of this form, we get

$$
\begin{aligned}
& A C \varphi_{f_{1}}(C) \varphi_{2 f_{1}}(C) \cdots \varphi_{\left(m_{1}-1\right) f_{1}}(C) \varphi_{m_{1} f_{1}}(A)^{-1} \\
= & B D \varphi_{f_{2}}(D) \varphi_{2 f_{2}}(D) \cdots \varphi_{\left(m_{2}-1\right) f_{2}}(D) \varphi_{m_{2} f_{2}}(B)^{-1}
\end{aligned}
$$

and that both sides are reduced words.

Now either $|A| \leq|B|$ or $|B| \leq|A|$. Without loss of generality, assume that we are in the first case. Then $A$ is an initial segment of $B$ (i.e., $B=A B^{\prime}$ as a reduced word). Then by replacing $r, s_{1}$, and $s_{2}$ with $A^{-1} r A, A^{-1} s_{1} A$, and $A^{-1} s_{2} A$, we may assume that $A$ is trivial. To summarize the reductions we have made so far, we have

$$
\begin{aligned}
r & =\left(C, f_{1}\right)^{m_{1}}\left(\varepsilon, g_{1}\right)=(B, 0)\left(D, f_{2}\right)^{m_{2}}\left(\varepsilon, g_{2}\right)\left(B^{-1}, 0\right) \\
s_{1} & =\left(C, f_{1}\right)^{n_{1}}\left(\varepsilon, h_{1}\right) \\
s_{2} & =(B, 0)\left(D, f_{2}\right)^{n_{2}}\left(\varepsilon, h_{2}\right)\left(B^{-1}, 0\right) .
\end{aligned}
$$

The automorphisms $\varphi_{g_{1}}$ and $\varphi_{h_{1}}$ fix $C$, and the automorphisms $\varphi_{g_{2}}$ and $\varphi_{h_{2}}$ fix $D$ and $B$. Both sides of

$$
\begin{aligned}
& C \varphi_{f_{1}}(C) \varphi_{2 f_{1}}(C) \cdots \varphi_{\left(m_{1}-1\right) f_{1}}(C) \\
= & B D \varphi_{f_{2}}(D) \varphi_{2 f_{2}}(D) \cdots \varphi_{\left(m_{2}-1\right) f_{2}}(D) \varphi_{m_{2} f_{2}}(B)^{-1}
\end{aligned}
$$

are reduced words.

Now we will show that either $m_{1}=1$ or $B$ is trivial. Suppose that $B$ was non-trivial, say $B=b B^{\prime}$. First note that the length of $C$ is greater than one, as otherwise $C=b$ and $\varphi_{\left(m_{1}-1\right) f_{1}}(C)=\varphi_{m_{2} f_{2}}\left(b^{-1}\right)$; but there is no $e \in \mathcal{H} / \mathcal{R}$ such that $\varphi_{e}(b)=b^{-1}$. Then we must have $C=b C^{\prime} \varphi_{m_{2} f_{2}-\left(m_{1}-1\right) f_{1}}\left(b^{-1}\right)$ for some $C^{\prime}$. We have $m_{1} f_{1}+g_{1}=m_{2} f_{2}+g_{2}$. Since $b$ appears both in $C$ and in $B$, it is fixed by both $\varphi_{g_{1}}$ and $\varphi_{g_{2}}$. Thus $C=b C^{\prime} \varphi_{f_{1}}\left(b^{-1}\right)$. But then if $m_{1}>1$,

$$
C \varphi_{f_{1}}(C) \varphi_{2 f_{1}}(C) \cdots \varphi_{\left(m_{1}-1\right) f_{1}}(C)
$$


is not a reduced word. So we conclude that either $m_{1}=1$ or $B$ is trivial.

Case 1. Suppose that $m_{1}=1$.

We have

$$
r=\left(C, f_{1}\right)\left(\varepsilon, g_{1}\right)=(B, 0)\left(D, f_{2}\right)^{m_{2}}\left(\varepsilon, g_{2}\right)\left(B^{-1}, 0\right) .
$$

Also, as reduced words,

$$
C=B D \varphi_{f_{2}}(D) \varphi_{2 f_{2}}(D) \cdots \varphi_{\left(m_{2}-1\right) f_{2}}(D) \varphi_{m_{2} f_{2}}(B)^{-1} .
$$

Since the right hand side is a reduced word, $\varphi_{g_{1}}$ and $\varphi_{h_{1}}$ fix $B$ and $D$ since each letter in $B$ and $D$ appears in $C$. Thus

$$
s_{1}=\left(C, f_{1}\right)^{n_{1}}\left(\varepsilon, h_{1}\right)=\left[(B, 0)\left(D, f_{2}\right)^{m_{2}}\left(B^{-1}, 0\right)\left(\varepsilon, f_{1}-m_{2} f_{2}\right)\right]^{n_{1}}\left(\varepsilon, h_{1}\right) .
$$

Now $f_{1}+g_{1}=m_{2} f_{2}+g_{2}$. Since $\varphi_{g_{1}}$ and $\varphi_{g_{2}}$ fix $B$ and $D, \varphi_{f_{1}-m_{2} f_{2}}$ also fixes $B$ and $D$. Thus

$$
s_{1}=(B, 0)\left(D, f_{2}\right)^{m_{2} n_{1}}\left(\varepsilon, h_{1}+n_{1}\left(f_{1}-m_{2} f_{2}\right)\right)\left(B^{-1}, 0\right)
$$

and $h_{1}+n_{1}\left(f_{1}-m_{2} f_{2}\right)$ fixes $D$. Thus $s_{1}$ and $s_{2}$ commute. This is a contradiction.

Case 2. $B$ is trivial.

Let $|C|=k$ and $|D|=\ell$. Suppose without loss of generality that $k \geq \ell$. Let $d_{0}, d_{1}, d_{2}, \ldots$ be the reduced word

$$
C \varphi_{f_{1}}(C) \varphi_{2 f_{1}}(C) \cdots \varphi_{\left(m_{1}-1\right) f_{1}}(C)=D \varphi_{f_{2}}(D) \varphi_{2 f_{2}}(D) \cdots \varphi_{\left(m_{2}-1\right) f_{2}}(D) .
$$

Then we have

$$
\begin{aligned}
d_{i} & =\varphi_{f_{2}}\left(d_{i-\ell}\right) & & \text { for } i \geq \ell \\
\varphi_{\left(m_{1}-1\right) f_{1}}\left(d_{k-\ell+i}\right) & =\varphi_{\left(m_{2}-1\right) f_{2}}\left(d_{i}\right) & & \text { for } 0 \leq i<\ell
\end{aligned}
$$

Let $e=\operatorname{gcd}(k, \ell)$.

Given $p, q \geq 0$, write $i=q \ell-p k+r$ with $0 \leq r<e$ and assume that $0 \leq i<$ $m_{1} k=m_{2} \ell$. Note that every $i, 0 \leq i<m_{1} k=m_{2} \ell$, can be written in such a way. We claim that

$$
d_{i}=\varphi_{q f_{2}+p\left[\left(m_{1}-1\right) f_{1}-m_{1} f_{2}\right]}\left(d_{r}\right) .
$$

We argue by induction, ordering pairs $(q, p)$ lexicographically. For the base case $p=q=0$ we note that $d_{r}=\varphi_{0}\left(d_{r}\right)$. If $\ell \leq i$, then we must have $q>0$. By the induction hypothesis, $d_{i-\ell}=\varphi_{(q-1) f_{2}+p\left[\left(m_{1}-1\right) f_{1}-m_{2} f_{2}\right]}\left(d_{r}\right)$. So

$$
d_{i}=\varphi_{f_{2}}\left(d_{i-\ell}\right)=\varphi_{q f_{2}+p\left[\left(m_{1}-1\right) f_{1}-m_{2} f_{2}\right]}\left(d_{r}\right) .
$$

If $0 \leq i<\ell$, and $(q, p) \neq(\varepsilon, 0)$, then $q>0$ and $p>0$. Note that

$d_{k-\ell+i}=\varphi_{(q-1) f_{2}+(p-1)\left[\left(m_{1}-1\right) f_{1}-m_{2} f_{2}\right]}\left(d_{r}\right)=\varphi_{\left(q f_{2}+p\left[\left(m_{1}-1\right) f_{1}-m_{2} f_{2}\right]-\left[\left(m_{1}-1\right) f_{1}-\left(m_{2}-1\right) f_{2}\right]\right.}\left(d_{r}\right)$

by the induction hypothesis and so

$$
d_{i}=\varphi_{\left(m_{1}-1\right) f_{1}-\left(m_{2}-1\right) f_{2}}\left(d_{i+k-\ell}\right)=\varphi_{q f_{2}+p\left[\left(m_{1}-1\right) f_{1}-m_{2} f_{2}\right]}\left(c_{r}\right) .
$$

This completes the induction.

Write $e=q \ell-p k$ with $p, q \geq 0$. Let $f=q f_{2}+p\left[\left(m_{1}-1\right) f_{1}-m_{2} f_{2}\right]$. Then each $i, 0 \leq i<k m_{1}$, can be written as $i=s e+r$ with $0 \leq r<d$, and so

$$
d_{i}=\varphi_{s f}\left(d_{r}\right) .
$$

Let $E=d_{1} \cdots d_{e}$. Then

$$
C=E \varphi_{f}(E) \cdots \varphi_{\left(\frac{k}{e}-1\right) f}(E)
$$


Similarly,

$$
D=E \varphi_{f}(E) \cdots \varphi_{\left(\frac{\ell}{e}-1\right) f}(E) .
$$

Also,

$$
\varphi_{f_{1}}(E)=d_{k} \cdots d_{k+e-1}=\varphi_{\frac{k}{e} f}\left(d_{0}, \ldots, d_{e-1}\right)=\varphi_{\frac{k}{e} f}(E)
$$

and

$$
\varphi_{f_{2}}(E)=d_{\ell} \cdots d_{\ell+e-1}=\varphi_{\frac{\ell}{e} f}\left(d_{0}, \ldots, d_{e-1}\right)=\varphi_{\frac{\ell}{e} f}(E) .
$$

So $\varphi_{f_{1}}(C)=\varphi_{\frac{k}{e} f}(C)$ and $\varphi_{f_{2}}(D)=\varphi_{\frac{\ell}{e} f}(D)$. Hence

$$
s_{1}=\left(C, f_{1}\right)^{m_{1}}\left(\varepsilon, h_{1}\right)=(E, f)^{\frac{m_{1} k}{e}}\left(\varepsilon, h_{1}+m_{1} f_{1}-\frac{m_{1} k}{e} f\right)
$$

and

$$
s_{2}=\left(D, f_{2}\right)^{m_{1}}\left(\varepsilon, h_{2}\right)=(E, f)^{\frac{m_{2} \ell}{e}}\left(\varepsilon, h_{2}+m_{2} f_{2}-\frac{m_{2} \ell}{e} f\right)
$$

Note that $\varphi_{h_{1}}$ and $\varphi_{h_{2}}$ both fix $E$, since they fix $C$ and $D$ respectively. Also, since $\varphi_{f_{1}}(E)=\varphi \frac{k}{e} f(E), \varphi_{m_{1} f_{1}-\frac{m_{1} k}{e} f}$ fixes $E$. Similarly, $\varphi_{m_{2} f_{2}-\frac{m_{2} \ell}{e} f}$ fixes $E$. So $s_{1}$ and $s_{2}$ commute. This is a contradiction.

Lemma 22. Fix $r \in \mathcal{G}$. If $r^{2} \in \mathcal{H} / \mathcal{R}$, then $r \in \mathcal{H} / \mathcal{R}$.

Proof. Write $r=(A, f)$. We will show that if $r \notin \mathcal{H} / \mathcal{R}$, i.e. if $A \neq \varepsilon$, then $r^{2} \notin \mathcal{H} / \mathcal{R}$. Since

$$
r^{2}=\left(A \varphi_{f}(A), 2 f\right)
$$

we must show that $A \varphi_{f}(A)$ is non-trivial. Suppose that it was trivial; then the length of $A$ as a reduced word must be even. (If the length of $A$ was odd, say $A=A_{1} a A_{2}$ with $A_{1}$ and $A_{2}$ of equal lengths, then

$$
A \varphi_{f}(A)=A_{1} a A_{2} \varphi_{f}\left(A_{1}\right) \varphi_{f}(a) \varphi_{f}\left(A_{2}\right)=\varepsilon .
$$

So it must be that $\varphi_{f}(a)=a^{-1}$, which cannot happen for any letter a.) Write $A=B C$, where $B$ and $C$ are each half the length of $A$. Then since $A \varphi_{f}(A)$ is the trivial word, $C \varphi_{f}(B)$ is the trivial word; thus $C=\varphi_{f}\left(B^{-1}\right)$. So $A=B \varphi_{f}\left(B^{-1}\right)$, and

$$
A \varphi_{f}(A)=B \varphi_{f}\left(B^{-1}\right) \varphi_{f}(B) \varphi_{2 f}\left(B^{-1}\right)=B \varphi_{2 f}\left(B^{-1}\right) .
$$

Since $A \varphi_{f}(A)$ is the trivial word, $\varphi_{2 f}(B)=B$. Since $A$ is not the trivial word, $B \neq \varphi_{f}(B)$. But this is impossible, as $p_{i}, q_{i}$, and $r_{i}$ were all chosen to be odd primes.

The next lemma is the heart of the existential definition of $(\mathcal{H} / \mathcal{R})^{\mathcal{G}}$. The proof is to show that under the hypotheses of the lemma, elements not in $(\mathcal{H} / \mathcal{R})^{\mathcal{G}}$ such as in Lemma 21 must exist.

Lemma 23. Let $r, s_{1}, s_{2}, s_{3} \in \mathcal{G}$. Suppose that $r$ commutes with $s_{1}, s_{2}$, and $s_{3}$, but that no two of $s_{1}, s_{2}$, and $s_{3}$ commute. Then $r \in(\mathcal{H} / \mathcal{R})^{\mathcal{G}}$.

Proof. If at least two of $s_{1}, s_{2}$, and $s_{3}$ are not in $(\mathcal{H} / \mathcal{R})^{\mathcal{G}}$, then this follows immediately by Lemma 21. Otherwise, without loss of generality suppose that $s_{1}$ and $s_{2}$ are in $(\mathcal{H} / \mathcal{R})^{\mathcal{G}}$. By Lemma 12, $s_{1} s_{2} \notin(\mathcal{H} / \mathcal{R})^{\mathcal{G}}$.

Note that $r$ commutes with $s_{1} s_{2}$ and with $s_{1}\left(s_{2}\right)^{2}$. Also, $s_{1} s_{2}$ does not commute with $s_{1}\left(s_{2}\right)^{2}$, since if it did, then

$$
s_{1} s_{2} s_{1} s_{2} s_{2}=s_{1} s_{2} s_{2} s_{1} s_{2} \Rightarrow s_{1} s_{2}=s_{2} s_{1} .
$$


We claim that $s_{1}\left(s_{2}\right)^{2} \notin(\mathcal{H} / \mathcal{R})^{\mathcal{G}}$. If $s_{1}\left(s_{2}\right)^{2}$ was in $(\mathcal{H} / \mathcal{R})^{\mathcal{G}}$, then by Lemma 12 , we could write

$$
s_{1}=(A, 0)(\varepsilon, g)\left(A^{-1}, 0\right) \text { and }\left(s_{2}\right)^{2}=(A, 0)(\varepsilon, h)\left(A^{-1}, 0\right) .
$$

Then let $s_{2}^{\prime}=\left(A^{-1}, 0\right) s_{2}(A, 0)=(C, f)$. Then $\left(s_{2}^{\prime}\right)^{2}=(\varepsilon, h)$, and so by Lemma 22 , $s_{2}^{\prime}=(\varepsilon, f)$. Thus $s_{2}=(A, 0)(\varepsilon, f)\left(A^{-1}, 0\right)$. So $s_{1}$ and $s_{2}$ would commute; since we know that $s_{1}$ and $s_{2}$ do not commute, $s_{1}\left(s_{2}\right)^{2} \notin(\mathcal{H} / \mathcal{R})^{\mathcal{G}}$.

By Lemma 21, with $r, s_{1} s_{2}$, and $s_{1} s_{2}^{2}$, we see that $r$ is in $(\mathcal{H} / \mathcal{R})^{\mathcal{G}}$.

The existential definition of $(\mathcal{H} / \mathcal{R})^{\mathcal{G}}$ comes from the previous lemma. It remains only to show that if $r \in(\mathcal{H} / \mathcal{R})^{\mathcal{G}}$, then the hypothesis of the previous lemma is satisfied.

Proof of Lemma 11. By the previous lemma, it suffices to show that if $r \in(\mathcal{H} / \mathcal{R})^{\mathcal{G}}$, then there are $s_{1}, s_{2}$, and $s_{3}$ such that $r$ commutes with $s_{1}, s_{2}$, and $s_{3}$, but no two of these commute with each other. If $r=(A, 0)(\varepsilon, g)\left(A^{-1}, 0\right)$, let $s_{1}=$ $(A, 0)\left(u_{0}, 0\right)\left(A^{-1}, 0\right), s_{2}=(A, 0)\left(u_{1}, 0\right)\left(A^{-1}, 0\right)$, and $s_{3}=(A, 0)\left(u_{2}, 0\right)\left(A^{-1}, 0\right)$. Then $r$ commutes with $s_{1}, s_{2}$, and $s_{3}$ since $g$ fixes $u_{0}, u_{1}$, and $u_{2}$, but no two of $s_{1}, s_{2}$, and $s_{3}$ commute with each other as $u_{0}, u_{1}$, and $u_{2}$ do not commute with each other.

\section{REFERENCES}

[Ber90] G. M. Bergman. Ordering coproducts of groups and semigroups. J. Algebra, 133(2):313$339,1990$.

[DK86] R. G. Downey and S. A. Kurtz. Recursion theory and ordered groups. Ann. Pure Appl. Logic, 32(2):137-151, 1986.

[Dob83] V. P. Dobritsa. Some constructivizations of abelian groups. Sibirsk. Mat. Zh., 24(2):18$25,1983$.

[DR00] R. G. Downey and J. B. Remmel. Questions in computable algebra and combinatorics. In Computability theory and its applications (Boulder, CO, 1999), volume 257 of Contemp. Math., pages 95-125. Amer. Math. Soc., Providence, RI, 2000.

[KM96] V. M. Kopytov and N. Ya. Medvedev. Right-ordered groups. Siberian School of Algebra and Logic. Consultants Bureau, New York, 1996.

[LS01] R. C. Lyndon and P. E. Schupp. Combinatorial group theory. Classics in Mathematics. Springer-Verlag, Berlin, 2001. Reprint of the 1977 edition.

[Shi47] H. Shimbireva. On the theory of partially ordered groups. Rec. Math. [Mat. Sbornik] N.S., 20(62):145-178, 1947.

[Sol02] R. Solomon. $\Pi_{1}^{0}$ classes and orderable groups. Ann. Pure Appl. Logic, 115(1-3):279-302, 2002 .

[Vin49] A. A. Vinogradov. On the free product of ordered groups. Mat. Sbornik N.S., 25(67):163$168,1949$. USA

Group in Logic and the Methodology of Science, University of California, Berkeley,

E-mail address: matthew.h-t@berkeley.edu

$U R L:$ www.math. berkeley.edu/ mattht 